\author{
Hydrodynamics VI-Theory and Applications - Cheng \& Yeow (eds) \\ o 2005 Taylor \& Francis Group, London, ISBN 0415363047
}

\title{
Vortex dislocation in wake-type flow caused by local spanwise nonuniformity
}

\author{
G. C. Ling \\ LNM, Institute of Mechanics, Chinese Academy of Sciences, Beijing, China
}

H. L. Zhao

RCFD, College of Science, PLA University of Science and Technology, Nanjing, Jiangsu, China

\author{
J. Y. Niu \\ LHD, Institute of Mechanics, Chinese Academy of Sciences, Beijing, China
}

\begin{abstract}
DNS of spatiotemporal evolution of a wake-type flow is performed. In the incoming flow, a local spanwise nonuniformity in momentum defect is initially imposed. Results show that the spanwise nonuniformity leads to a series of symmetric twist vortex dislocation in downstream of the flow. Vortex line variations and substantial transition of vorticity from spanwise to the streamwise and vertical directions clearly feature the generation of a vortex dislocation and the real vortex linking in the dislocation. Dynamical process and the mechanism responsible for the vortex dislocation are described.
\end{abstract}

\section{INTRODUCTION}

A number of previous works have devoted to study of wake three dimensionalities and shown that for low Reynolds numbers the vortex dislocation is a fundamental characteristic of bluff body wakes. Depending on the three-dimensional flow conditions, the vortex dislocation may present various modes or patterns, for instance, vortex “splitting and reconnection”, vortex “kinks” or "holes", or a kind of large-scale spot-like vortex dislocations and so forth. Those phenomena can be found in cylinder wakes with diameter variation or nonuniform coming flows, or in the wake of stepped cylinder, as well as in the wake of the cylinder with end effects. Detailed presentations of them can be found in the experimental studies and reviews of Gerrard (1996), Bearman (1992), Lewis \& Gharib (1992), Eisenlohr \& Eckelmann (1989), Williamson (1992, 1996), Papangelou (1992) and many others. Again, the vortex dislocations may be characterized by "a local break of continuity appearing on the "spinal column" of a main vortex row" , which occurs randomly in the natural transition of cylinder wakes (Braza \& Persillson 2001). Also, a kind of vortex "branching interaction" was observed in transition of mixing layer (Dallard \& Browand 1993). The occurrence of vortex dislocations is closely related to the break-up to turbulence and is considered a new mechanism of the flow transition. Moreover, it is associated with the variation of forces acting on the cylinders. Therefore, study of the vortex dislocation has received a great deal of attention.

However, due to its highly three-dimensionality and very complex in structure, the detailed information and features of vortex linkages in vortex dislocations are still not yet known thoroughly. Dynamics related is not fully understood. Mechanism explanation responsible for the vortex dislocation in previous studies was basically based on an inviscid vortex dynamics, it is difficult to offer a complete understanding for the real viscous flows. It is believed that to make further numerical study to address those issues is significant. 
In present study DNS of spatial-temporal evolution of a typical wake-type flow with a local spanwise nonuniformity is performed. The nonlinear developments of the flow instability and nonuniform evolution of the flow are described by numerical results. A clear identification of scenario of the formation of the vortex dislocation and the basic feature of the complex vortex linkage in it are reported by analyzing mainly the substantial modification of vorticity field and vortex lines tracks. Therefore a full understanding of the dislocation dynamics, even the definition of the technical terms of vortex dislocation is gained. Detailed information on how wake flow behaviors accommodate the difference in phase, frequency and strength of vortices between shedding cells via vortex dislocation is given now. The influence of different nonuniformity in the coming flow on vortex dislocation and the feature of the transition behaviors are also investigated in present work. However, due to the space limitation of the present paper, those results will be reported in another paper.

\section{NUMERICAL METHODS}

In present calculations, the velocity profile of incoming flow is taken as

$$
U(y, z)=1.0-a(z)\left(2.0-\cosh (b y)^{2}\right) e^{-(c y)^{2}}
$$

which represents the time average streamwise velocity profile in the cylinder near wake where the flow is most unstable. $a(z)=1.1+0.4 \exp \left(-z^{2}\right)$ is imposed to simulate a local nonuniformity in momentum defect, which decays with the increase of spanwise distance. $b$ and $c$ are parameters determined by referring DNS solution of Kaniadakis \& Triantafyllou (1992), as well as an experimental measurement in cylinder wake by Nishioka \& Sato (1974). So this kind of wake-type flow has some similar behavior, to certain extent, to the wake behind a cylinder with a local small disturbance in diameter. It is helpful for understanding the nature of vortex dislocation.

DNS of the wake flow evolution has carried out by solving three-dimensional Nervier-Stokes equations using the compact finite difference-Fourier Spectral hybrid method. A detailed presentation of these equations and the numerical method can be found in Xiong \& Ling (1996) and Ling \& Xiong (2001). In the method the periodic boundary condition in spanwise direction is assumed. The $m$-th Fourier component of Navier-Stokes equations is

$$
\frac{\partial \vec{u}_{m}}{\partial t}+F_{m}[(\vec{u} \cdot \nabla) \vec{u}]=-\nabla_{m} p_{m}+\frac{1}{\operatorname{Re}} \nabla_{m}^{2} \vec{u}_{m}
$$

For time discretization of the equation a third order mixed explicit-implicit schemes in used. The solution procedure for these $\mathrm{m}-$ th harmonic equations is split into the following three substeps: 


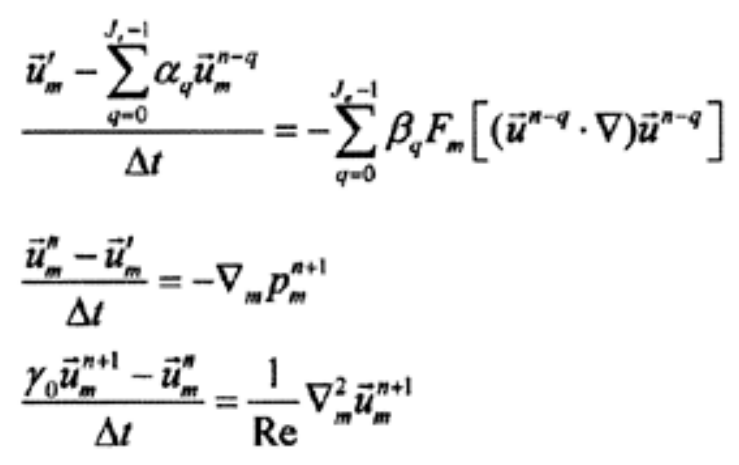

(3)

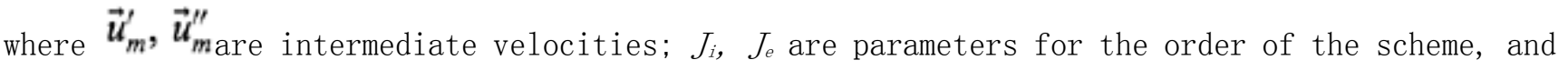
$a_{q}, \beta_{q}, y_{0}$ are appropriately chosen weights. To evaluate the nonlinear terms in the split equations, the pseudo-spectral method is adopted, and the fifth order upwind compact scheme is used to approximate the terms. For solving Helmholtz equations for pressure and velocity, a nine-point compact scheme of forth-order accuracy is derived and sixth-order central compact scheme is used

for non-homogeneous term calculations. For the details of the parameter values in the equation (3) and the particular representation of numerical schemes, readers may refer to Ling \& Xiong (2001). For the boundary conditions a semi-discretized pressure boundary conditions and a generalized nonreflecting-type outflow boundary conditions are used (Xiong \& Ling 1996). Reynolds number is defined as $\operatorname{Re}=U_{0} D / \mathrm{V}$, here $U_{0}, D$ represent an infinite potential flow velocity and characteristic length, respectively. The computation domain is 100, 30, 30(D) in streamwise, transverse and spanwise directions, respectively. The cutoff of the truncated Fourier series is $N=64$, and the grid points in $x^{-} y$ plane are $202 \times 62$. The numerical code used in the present work has been verified first. It shows that evolution of the wake-type flow without the local spanwise non-uniformity results in a normal Kármán vortex street with a Strouhal number of 0.189 , which is well compared with both numerical simulation of wake-type flow evolution ( $\mathrm{St}=0.195$, see Karniadakis \& Triantafyllou, 1992) and DNS results of flow around cylinder ( $\mathrm{St}=0.179$, see Triantafyllou \& Karniadakis, 1990).

\section{NUMERICAL RESULTS}

Numerical results exhibit that a series of symmetric twist vortex dislocations are generated in the middle downstream as shown figure 1, where we can also see the streamwise and vertical vorticity branches connected to the large distorted spanwise vortex rolls. Isosurface of vorticity, vorticity component contours and fluctuating velocity distributions are used to describe the generation and the configuration of vortex dislocations. However the details of vortex linkages in the vortex dislocations, especially the connections with opposite sign vortices are still unclear. It can not be identified from those isosurface patterns. For understanding the real spatial linking and their dynamic process, some representative vorticity lines emitted from different part of a vortex roll located at different downstream positions are investigated. Their direction and tracks describe clearly how the vortex dislocations generate and what the real vortex linkages are in the vortex dislocations. A sample is shown in figure 2 .

Results show that in the upstream region, the vortex lines start to undulate in the spanwise direction, then undergo a stronger distortion near the central area (fig. 2a). In the middle 
downstream, we can see a set of vortex lines pass across the span with a big distortion near the central area, and the others go into upstream adjacent vortex core, after turning 180 degree to upstream (fig. 2b). It is interesting to note that the vortex lines starting from the different vertical positions of the same vortex were shown in figure $2 \mathrm{c}$ will go back to downstream and join the next spanwise vortex. That means some parts of the positive vortex are connected with adjacent negative vortices, as if they are "split" from that positive vortex. The connection between the positive vortex and its adjacent negative vortex takes place by a cross-vortex street mode.

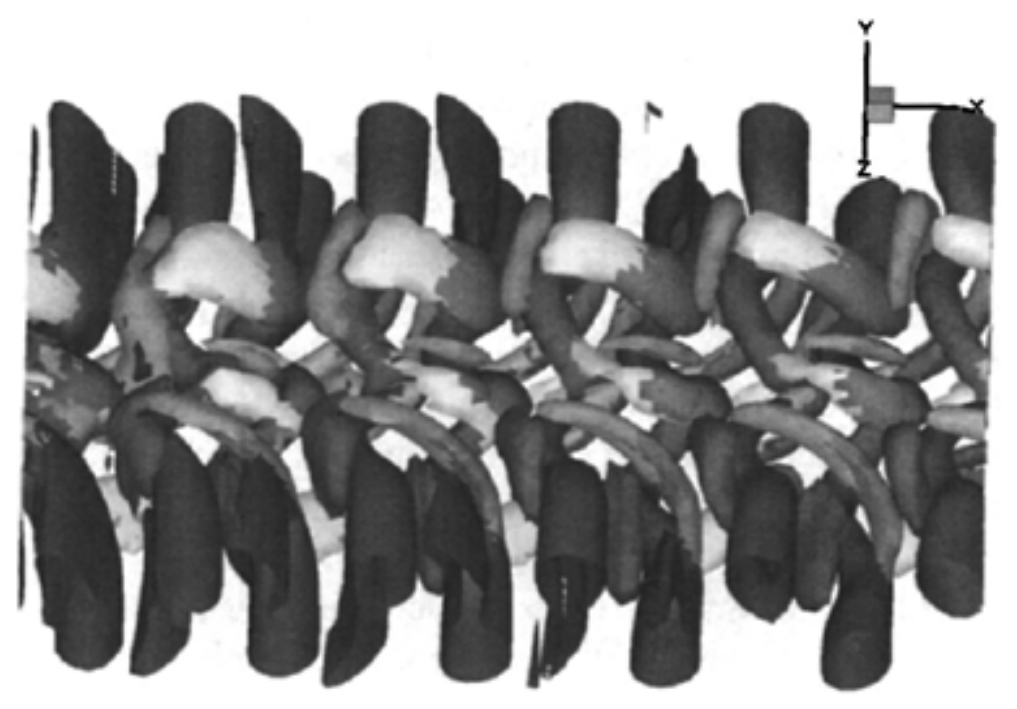

Figure 1. Isosurface of $\omega_{z}= \pm 0.12, \omega_{x}= \pm 0.1$ and $\omega_{y}= \pm 0.06$ in the middle down stream region at $t=300.0$. 


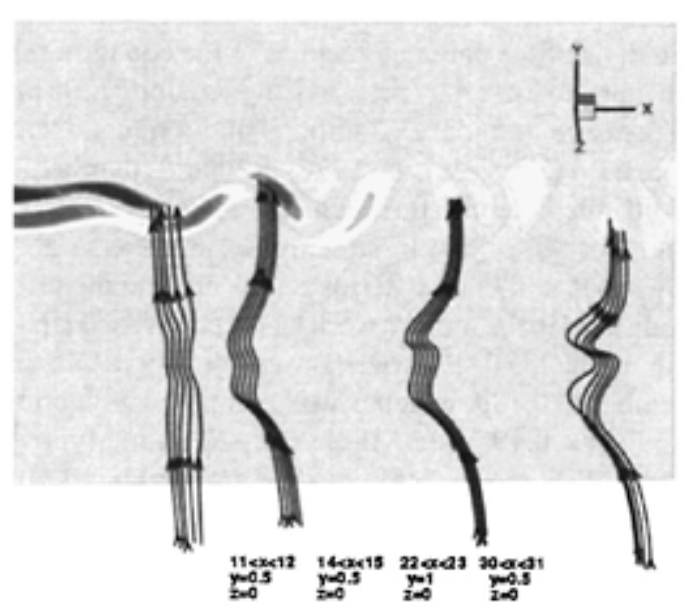

(a)

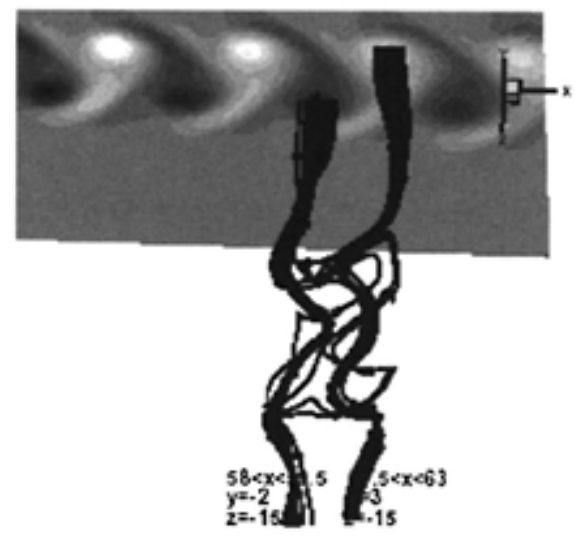

(b)

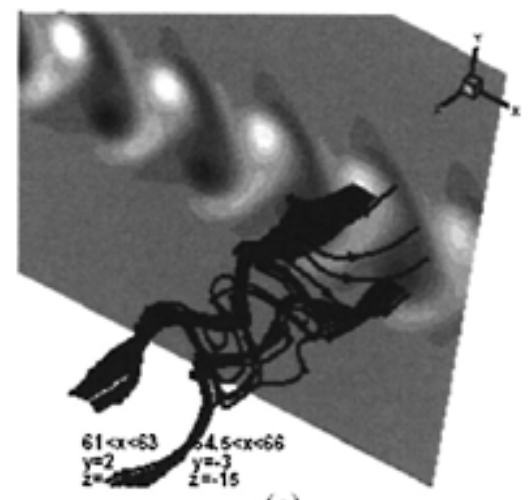

(c)

Figure 2. Vortex lines with different beginning or ending positions. The background is the counter of $\omega_{z}$ on the plane $z=-15$.

Thus the basic features of vortex linking in vortex dislocation can be briefly summarized as follows: 1) large undulated spanwise vortex rol1; 2) some split spanwise vortex lines diverting their direction and connecting with adjacent vortices by a cross-vortex street mode; 3) the distorted vortex lines wind round their neighboring spanwise vortices. From above results it is clear that the generation of vortex dislocation is a continuous process. In the period, the spanwise vortices, in some places of the flow field, undergo a substantial modification from its original direction to the streamwise and, meantime, to the vertical direction. The phenomenon of "vortex split" or "reconnection" does not occur instantly or abruptly. The phenomenon "break of continuity appearing on vortex spinal column" in a real viscous flow does not exist, it is just the vorticity transition both in direction and in quantity from original spanwise to others.

Figure 3 is a spanwise vorticity distribution along span. It clearly shows that the vortex street production along the spanwise direction is nonsynchronous. The initial phase and the strength of vortices between shedding cells are variation with the span. These spatial nonuniformities lead to occurrence of the vortex dislocations. From present calculation it is found that the appearance of the streamwise vorticity component and the vertical one are at some places where the rate of the phase variation along the span, reaches a maximum value, and the three-dimensional vortex dislocation structure appears. This feature also gives us some idea on the mechanism for the vortex dislocations. 


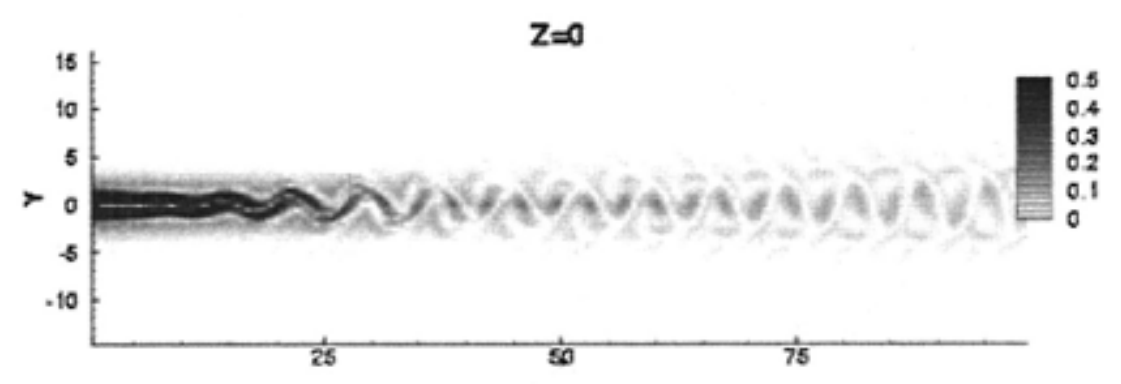

$Z=5.5$
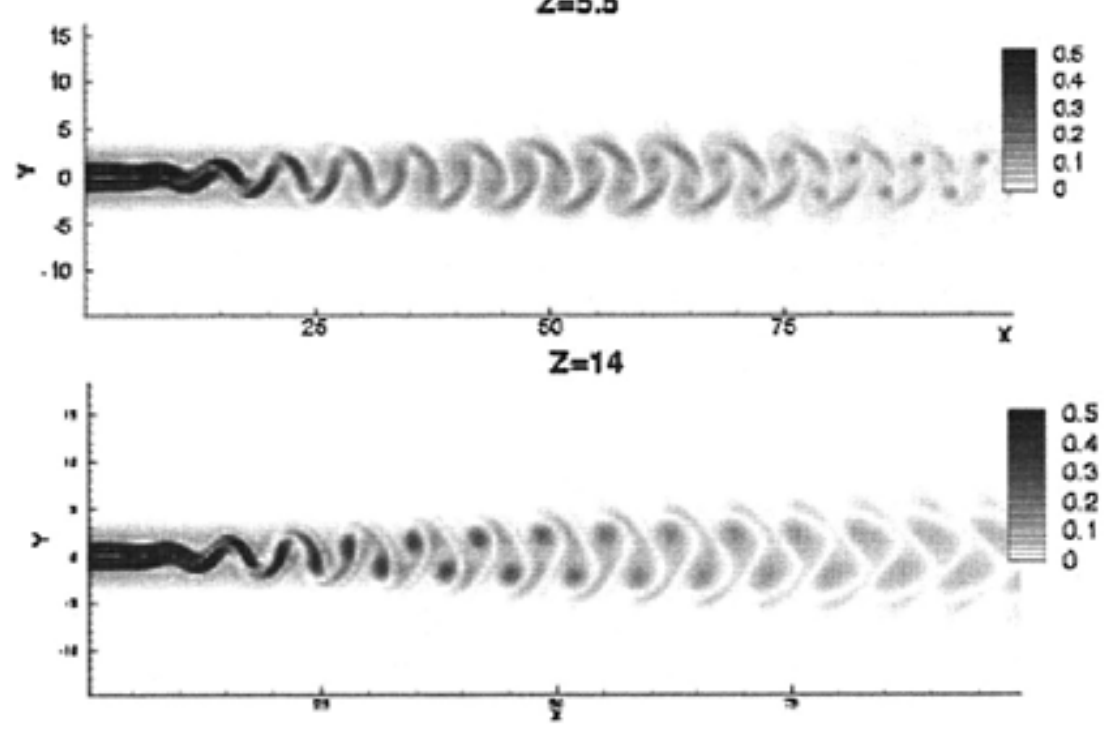

Figure 3. Spanwise vorticity distribution at different spanwise locations at $t=260$.

In our calculations frequency spectrum characteristics, low frequency modulation, wavelet analysis of velocity fluctuations are obtained. The irregular changes of frequency, vorticity and velocity at different locations caused by the vortex dislocations are analyzed in detail. The irregularity is closely related to the break-up to the local turbulence and the spatial chaos of the flow. The transition features of the wake-type flow are described.

\section{CONCLUDING REMARKS}

From DNS of the wake-type flow evolution, by carefully tracing the direction variations of the vorticity lines and their track features, the generation of vortex dislocations and the vortex linkages in dislocations are clearly described. The basic aspects of a vortex dislocation in the present flow condition are also reported. These results are helpful to well understanding the phenomena of dislocation, the dynamics as well as the mechanism for vortex dislocation.

\section{ACKNOWLEDGEMENT}

This work is supported by the Special Funds of Major State Basic Research Projects under Grant No. G1999032801, National Natural Science Foundation of China under Grant No 10272104, and partial1y supported by CAS INOVATION PROJECT.

\section{REFERENCES}


Bearman, P.W. 1992 Challenging problems in bluff body wakes. Bluff-Body Wakes, Dynamics and Instabilities, IUTAM Symposium. Göttingen: Springer-Verlag.

Braza, M., Faghani, D. \& Persillson, H. 2001 Successive Stages and the Role of Natural Vortex Dislocation in Three-dimensional Wake Transition. J. Fluid Mech. 439:1-41.

Dallard, T. \& Browand, F. K. 1993 The Growth of Large Scales at Defect Sites in the Plane Mixing Layer. J. Fluid Mech. 247:339-368.

Eisenlohr, H. \& Eckelemann, H. 1989 Vortex splitting and its consequences in vortex street wake of cylinders at low Reynolds number. Phys. Fluid A 1:189-192.

Gerrard, J.H. 1966 The three-dimensional structure of the wake of a circular cylinder. J. Fluid Mech. 25:143-164.

Karniadakis, G.E. \& Triantafyllou, G.S. 1992 Three-dimensional Dynamics and Transition to Turbulence in the Wake of Bluff Objects. J. Fluid Mech. 238:1-30.

Lewis, C. G. \& Gharib, M. 1992 An Exploration of Wake Three-Dimensionalities Caused by a Local Discontinuity in Cylinder Diameter. Phys. Fluids A 4:104-117.

Ling, G. C. \& Xiong, Z.M. 2001 Generation of Large-scale Vortex Dislocations in a Three-dimensional Waketype Flow. Science in China (series A) 4(12):1585-1595.

Nishioka, M. \& Sato, H. 1974 Measurernents of Velocity Distributions in the Wake of a Circular Cylinder at Low Reynolds Numbers. J. Fluid Mech. 65:97-112.

Papangelou, A. 1992 Vortex shedding from slender cones at low Reynolds numbers. Bluff-Body Wakes, Dynamics and Instabilities, IUTAM Symposium. Göttingen: Springer-Verlag.

Triantafyllou, G. S. \& Karniadakis, G. E. 1990 Computational Reducibility of Unsteady Viscous Flow. Phys. Fluids A 2:653-658.

Williamson, C.H.K. 1992 The Natural and Forced Formation of Spot-like Vortex Dislocations in the Transition of a Wake. J. Fluid Mech. 243:393-441.

Williamson, C.H.K. 1996 Vortex Dynamics in the Cylinder Wake. Annu. Rev. Mech. 28:477-539.

Xiong, Z.M. \& Ling, G.C. 1996 Compact Finite Difference-Fourier Spectral Method for Three-dimensional Incompressible Navier-Stokes Equation. ACTA. Mechanica Sinica 12(4):296-306. 